GCA Technical Report No. 62-3-N

\title{
THEORETICAL ESTIMATES OF THE AVERAGE \\ SURFACE TEMPERATURE ON MARS
}

\author{
George Ohring \\ Wen Tang \\ Gloria DeSanto
}

TECHNICAL REPORT NO。 1

Contract No. NASw -286

Apri1 1962

GEOPHYSICS CORPORATION OF AMERICA

Bedford, Massachuset ts

$$
\text { prepared for }
$$

National Aeronautics Space Administration Washington, D. C. 


\begin{abstract}
Estimates of the average surface temperature on Mars are derived from radiative equilibrium considerations. A minimum possible surface temperature is estimated by computing the radiative cquilibrium temperature that the Martian surface :ould have if the planet had no atmosphere. An estimate of the maximum possible value of the average surface temperature is obtained by computing the surface temperature that would result from a maximum greenhouse mode1. The computations indicate that the average surface temperature is in the range $219 \mathrm{~K}$ to $233 \mathrm{~K}$. Comparisons of the theoretical computations with indications of surface temperature obtained from thermal emission obcervations are found to be in reasonable agreement.
\end{abstract}


TABLE OF CONTENTS

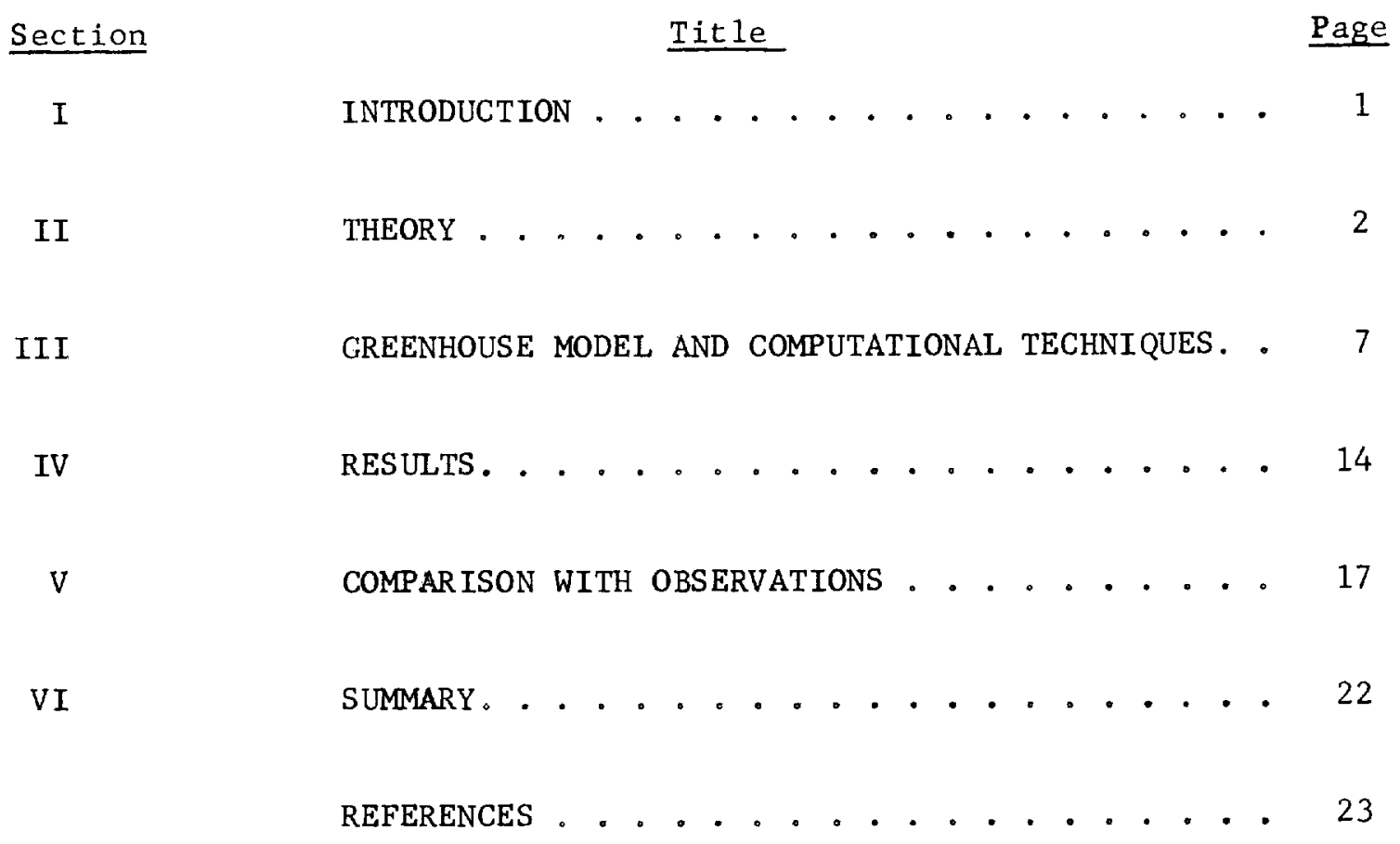




\section{THEORETICAL ESTIMATES OF THE AVERAGE \\ SURFACE TEMPERATURE ON MARS}

\section{INTRODUCTION}

Of extreme interest to meteorologists and astrophysicists who are involved in planetary atmospheres research is the average surface temperature of the planets. Planetary surface temperature can be derived from both experimental observation and theoretical reasoning. The observational determinations, which work well in the case of a rather tenuous atmosphere such as Mars', are based upon measurements of the planet's emission of infrared radiation; the theoretical determinations are based largely upon some sort of radiative equilibrium considerations. In the present study we derive estimates, based upon radiative equilibrium considerations, of the minimum and maximum possible value of the average temerature of the Martian surface. These estimates are then compared to some of the thermal emission observations. 


\section{THEORY}

The average surface temperature of a planet which has an atmosphere that is transparent to long wave radiation can be computed by equating the energy received from the sun to the energy emitted by the planets' surface. The following formula for the temperature of such a plant can be easily derived:

$$
\mathrm{T}^{4}=\frac{(\mathrm{S} \cdot \mathrm{C} \cdot)(1-A) \mu}{4 \sigma}
$$

where $\quad T$ is temperature,

S.c. is the solar constant at the earth ${ }^{8} \mathrm{~s}$ distance from the sun,

A is the planetary albedo,

$\sigma$ is the Stefan-Boltzmann constant, and

$\mu$ is a dilution factor equal to $r_{e}^{2} / r_{p}^{2}$,

where $r_{e}$ is the mean distance from the earth to the sun, and $r_{p}$ is the mean distance from the planet to the sun.

If one now introduces into such a planetary atmosphere, gases that absorb long wave radiation, a"green-house" effect is created and the average surface temperature will increase above that given by Equation (1). Thus, for an atmosphere that is not completely transparent to long wave radiation Equation (1) can be used to derive an estimate of the lowest possible value of the average planetary surface temperature. For the planet Mars the following numerical values can be substituted in Equation (1): 


$$
\begin{aligned}
A & =0.15 \\
\mu & =0.44 \\
s_{\triangle} c_{0} & =2.0 \mathrm{cal} \mathrm{cm}-2 \mathrm{~min}^{-1} \\
\sigma & =8.13 \times 10^{-11} \mathrm{cal} \mathrm{cm}^{-2} \mathrm{de \varepsilon}^{-4} \mathrm{~min}^{-1}
\end{aligned}
$$

With these substitutions the lowest possible value for the average planetary surface temperature for Mars turns out to be $219 \mathrm{~K}$.

The problem of estimating the maximum possible value of the average Martian surface temperature is more complex. We wish to maximize the green-house effect, within reason, in order to obtain a realistic estimate of the maximum surface temperature. The green-house effect depends essentially upon the amount and vertical distribution of absorbing gases, and on the vertical distribution of temperature. The greater the amount of absorbing gases - the more opaque the atmosphere is the greater will be the green whouse effect, and thus the higher will be the surface temperature. In our model (discussed later) we attempt to make realistic estimates of the maximum amounts of carbon dioxide, water vapor and ozone in the Martian atmosphere, and their distribution with height. Once the maximum amount of absorbing gas is fixed we have only to choose a realistic temperature distribution with height, which would maximize the surface temperature. For simplicity, and for lack of know ledge, we shall assume that the temperature variation with height is linear. This is probably a good approximation in the troposphere of Mars, as it is for the troposphere of the earth, and it is the troposphere rather than the upper atmosphere that contributes most to the radiation fluxes. The question we must now answer is: "What choice of 
constant lapse rate will produce a maximum green-house effect and thus a maximum surface temperature?" We can answer this question by going through the following qualitative reasoning。

The upward flux of long wave radiation at the top of Martian atmosphere must balance the incoming solar radiation that is not reflected back to space. This can be written as

$$
I_{0}(1-A)=W
$$

where $I_{0}$ is the average incoming flux of radiation at the top of the Martian atmosphere,

$A$ is the planetary a1bedo, and

$W$ is the upward flux of long wave raciation at the top of the atmosphere.

The upward flux $W$ is composed of radiation coming directly from the planet 's surface and of radiation emanating from the planet's atmosphere. If the atmosphere is isothermal, the radiation originating in the atmosphere will be at the same temperature as the radiation originating from the surface. Thus, the upward flux will remain constant with height and have a value equal to the black body flux emitted by the surface. The surface temperature of an isothermal atmosphere would then be the same as that computed from Equation (1), $i_{0} e_{0}, 219 \mathrm{~K}$. If the temperature decreases with height, however, the radiation emitted by the atmosphere would be at a lower temperature than that emitted by the ground. In order that $W$ now balance the incoming radiation, the surface temperature 
must be higher than $219 \mathrm{~K}$. The greater the lapse rate the greater is the surface temperature in order for balance to occur at the top of the atmosphere. In a planetary atmosphere the limiting lapse rate is the adiabatic lapse rate and we choose this as the lapse rate that will maximize the green house effect and hence the surface temperature。

With a given distribution of absorbers and temperature lapse rate, the outgoing radiation, $W$, is a function only of the surface temperature, and $c$ an be written as (1), (2)

$$
\begin{aligned}
& \mathrm{W}=\sigma \mathrm{T}_{\mathrm{s}}{ }^{4}-\int_{\mathrm{T}_{\mathrm{t}}}^{\mathrm{T}_{\mathrm{s}}} \mathrm{R}\left(\mathrm{CO}_{2}\right) \mathrm{dT}-\int_{\mathrm{T}_{t}}^{\mathrm{T} s} \mathrm{R}\left(\mathrm{H}_{2} \mathrm{O}\right) \mathrm{dT}-\int_{\mathrm{T}_{t}}^{\mathrm{T}_{\mathrm{t}}} \mathrm{R}\left(\mathrm{O}_{3}\right) \mathrm{dT} \\
& \text { where } \mathrm{R}=\int_{v_{1}}^{v_{2}} \frac{\partial \mathrm{v}}{\partial \mathrm{T}}\left(1-\tau_{f}\right) \mathrm{d} v
\end{aligned}
$$

and

$$
\begin{aligned}
& T_{s} \text { is surface temperature, } \\
& T_{t} \text { is the temperature at the top of the atmosphere } \\
& B \text { is the black body flux, } \\
& \tau_{f} \text { is the flux transmissivity, and } \\
& v \text { is frequency. }
\end{aligned}
$$

$R$ is a function of path length, $u_{2}$ and temperature, $T$, and the particular gas in question. Tables of $\mathrm{R}$ for carbon dioxide, water vapor and ozone have been presented by Elsasser ${ }^{(1)}$ and expanded by Hales, et al, $\mathrm{R}$ For carbon. dioxide covers the $15 \mu$ band, $\mathrm{R}$ for $\mathrm{H}_{2} \mathrm{O}$ covers the $6.3 \mu$ band, the window region, and the rotational band, and $\mathrm{R}$ for ozone covers the $9.6 \mu$ band. The integrals in Equation (3) can be evaluated numerically, given the temperature and absorber distribution in the atmosphere. It 
should be noted that both water vapor and carbon dioxide absorb radiation in the $15 \mu$ band. However, since we are attempting to maximize the green-house effect we shall treat these absorptions independently and make no correction for the overlap.

Equation (2) can now be written as:

$$
I_{0}(1-A)+\int_{T_{t}}^{T_{s}} R\left(C_{2}\right) d T+\int_{T_{t}}^{T} R\left(H_{2} \mathrm{O}\right) d T+\int_{T_{t}}^{T_{T}} R\left(0_{3}\right) d T=\sigma T_{s}^{4}
$$

To solve this equation for the surface temperature, $\mathrm{T}_{\mathrm{s}}$, one can assume a value for $T_{S}$ and perform the integrations on the left side of the equation. Upon adding the incoming radiation to the evaluated integrals, we can compute a second approximation of $\mathrm{T}_{\mathrm{s}}$ from Equation (5). This new value is then used to recalculate the integrals and obtain a third approximation, and so on, until a value of $\mathrm{T}_{\mathrm{S}}$ is obtained that balances Equation (5). The surface temperature obtained from this model represents an estimate of the maximum possible value of the average surface temperature on Mars. 


\section{III, GREEN-HOUSE MODEL AND COMPUTATIONAL TECHNIQUES}

The green-house model that we shall adopt is based upon reasonable estimates of the maximum amounts of carbon dioxide, water vapor and ozone in the atmosphere of Mars. Of these three constituents, carbon dioxide is the only one that has definitely been detected in are Mian atmosphere. Grandjean and Goody, ${ }^{(3)}$ in a re-analysis of Kuiper's (4) near infrared measurements, derive a carbon dioxide content of about $2 \%$ for the commonly accepted surface pressure of $85 \mathrm{mb}$. We shall simply double this value for our estimate of maximum carbon dioxide content. We shall further assume that all three gases are uniformly mixed with height so that the path lengths are a function only of pressure, and that a linear pressure correction can be applied directly to the path lengths as suggested by Elsasser。(1) With these assumptions and a carbon dioxide content of $4 \%$ the carbon dioxide path length for any pressure thickness is

$$
\Delta \mathrm{u}_{\mathrm{CO}_{2}}=86.8\left(\frac{\overline{\mathrm{p}}}{1000}\right) \Delta \mathrm{p}
$$

where $\Delta \mathrm{U}_{\mathrm{CO}_{2}}$ is the pressure corrected path length in a layer with mean pressure $\overline{\mathrm{p}} \mathrm{mb}$ and thickness $\Delta \mathrm{p}$.

Although water vapor has not been detected spectroscopically in the Martian atmosphere, the aqueous nature of the polar caps indicates that there must be some water vapor present. Dunham ${ }_{?}^{(5)}$ on the basis of spectroscopic observations, derived an upper 1 imit of 0.0015 times the 
terrestrial amount over Mt. Wilson on an average clear night. This is of the order of $10^{-3} \mathrm{~cm}$ of precipitable water with the terrestrial amount of about $0.7 \mathrm{~cm}$ prevailing during Dunham's observations. DeVaucouleurs (6) states that the amount of precipitable water is very likely much less than $4 \times 10^{-2} \mathrm{~cm}$ and probably less than $1 \times 10^{-2} \mathrm{~cm}$. We take as our estimate of the maximum water vapor content the value $10^{-2} \mathrm{~cm}$ of precipitable water. Assuming a constant mixing ratio with height, the pressure corrected water vapor path length for any pressure thickness can be written as

$$
\Delta \mathrm{u}_{\mathrm{H}_{2} \mathrm{O}}=1.18 \times 10^{-3}\left(\frac{\overline{\mathrm{p}}}{1000}\right) \Delta \mathrm{P}
$$

Ozone also has not been detected in the Martian atmosphere. An upper limit of $0.05 \mathrm{~cm}$ has been given by Kuiper ${ }^{(4)}$ who based his estimate on the failure of attempts to detect ozone by means of absorption spectra. Another estimate of the maximum amount of ozone has been derived by Marmo, et al, (7) who computed the total amount of photochemically produced ozone for an atmosphere that had a maximum possible amount of oxygen. The maximum amount of oxygen is about $2.5 \mathrm{~m}$ (Dunham ${ }^{(5)}$ ) and for this oxygen content Marmo, et al computed a value of about 0.15 $\mathrm{cm}$ for the total amount of ozone. Using this latter value as our estimate of the maximum amount of ozone on Mars we can express the pressure corrected ozone path lengths as

$$
\Delta \mathrm{u}_{3}=1.8 \times 10^{-2}\left(\frac{\overline{\mathrm{p}}}{1000}\right) \Delta \mathrm{p}
$$


As discussed previously the adiabatic lapse rate, approximately $3.7 \mathrm{deg} / \mathrm{km}_{2}$ is to be used in the radiation computations. The surface pressure is taken as $85 \mathrm{mb}$. In order to evaluate numerically the integrals appearing in Equation (5), the atmosphere is divided into nine layers of thickness $10 \mathrm{mb}$, except for the topmost layer, whose thickness is $5 \mathrm{mb}$. For example the integral for carbon dioxide is written as

$$
\sum_{i=1}^{9} R_{i}\left(\log \bar{u}_{i}, \bar{T}_{i}\right) \Delta T_{i}
$$

where $R_{i}$ is the $R$ value of the $i^{\text {th }}$ layer, which is a function of $\bar{u}_{i}$, the pressure corrected carbon dioxide path length from top of the atmosphere to the middle of the layer and $\bar{T}_{i}$, is the average temperature of the $i^{\text {th }}$ layer, and $\Delta T_{i}$ is the temperature difference between the bottom and top of the $i^{\text {th }}$ layer.

Mean temperatures and path lengths for each layer are used to obtain values of the $R$ function from Elsasser' $s$ (1) tables, and the computation is similar in all respects to that suggested by Elsasser except that numerical rather than graphical integration is used. In our model the temperature at the top of the atmosphere is zero $\mathrm{K}$ and the temperatures in the upper part of the atmosphere are below 193K. Since Elsasser's tables of $\mathrm{R}$ cover only the range $193 \leq \mathrm{T} \leq 313$, they were extrapolated

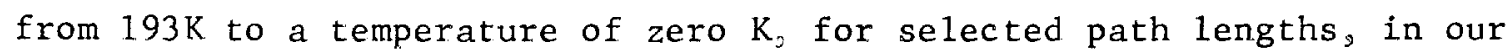
computations. The extrapolation to zero degrees $\mathrm{K}$ was performed with the knowledge that $R$ at zero degrees is equal to zero (see Equation (4)). As a check on the extrapolation, integrals of the form $\int_{0}^{193} R d T$ at 
constant path length were evaluated graphically and compared to similar integrals given by Elsasser. (1) Although there may still be some error in the values of $R$ used at temperatures less than $193 \mathrm{~K}$, these will not significantly affect the computations since the contributions of the upper layers to the outgoing radiation are much less than the contributions from the lower layers. Graphs showing the extrapolated $R$ curves are shown in Figures 1,2 , and 3. 


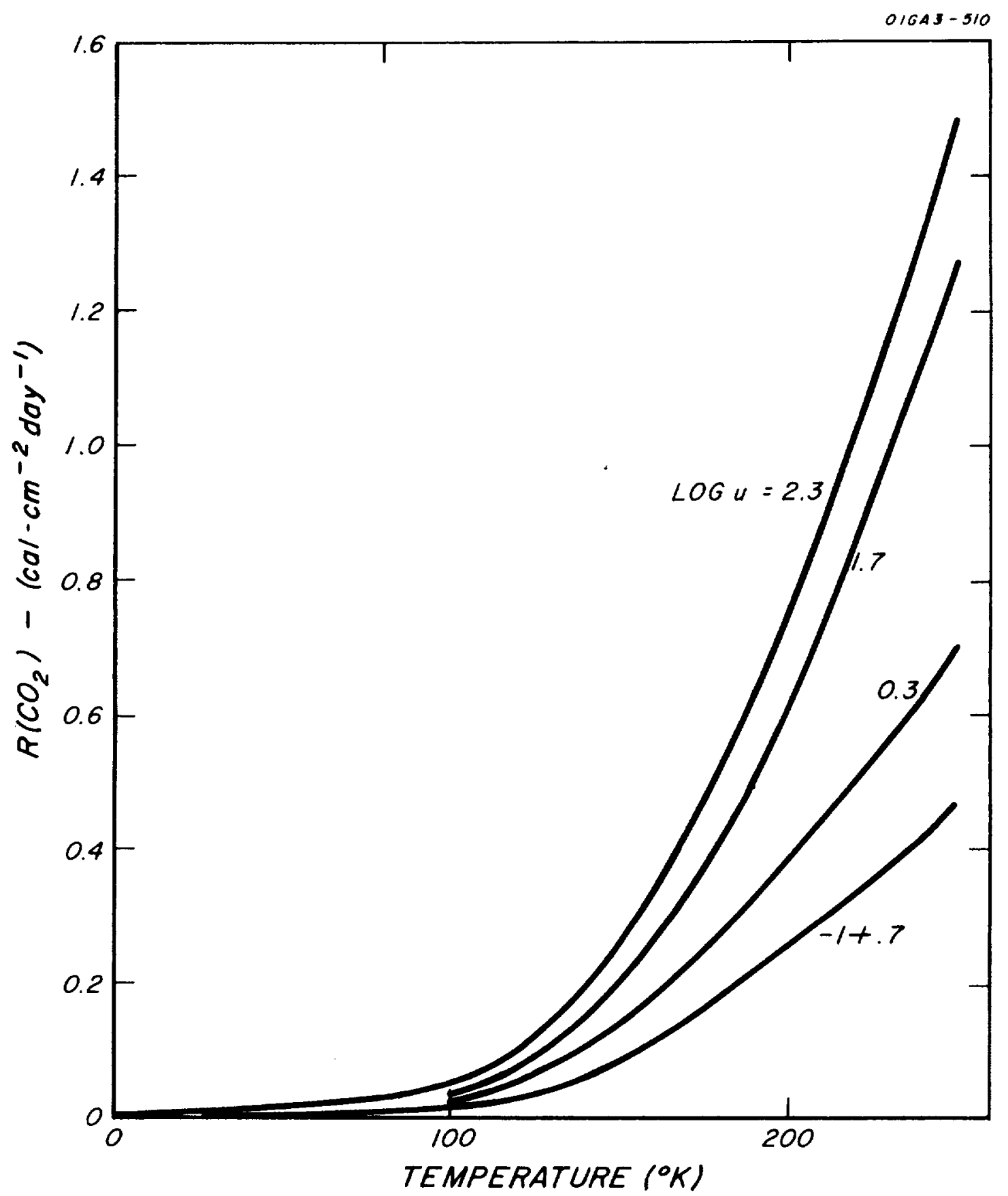

Figure 1. Extrapolated values of $\mathrm{R}\left(\mathrm{CO}_{2}\right)$. 


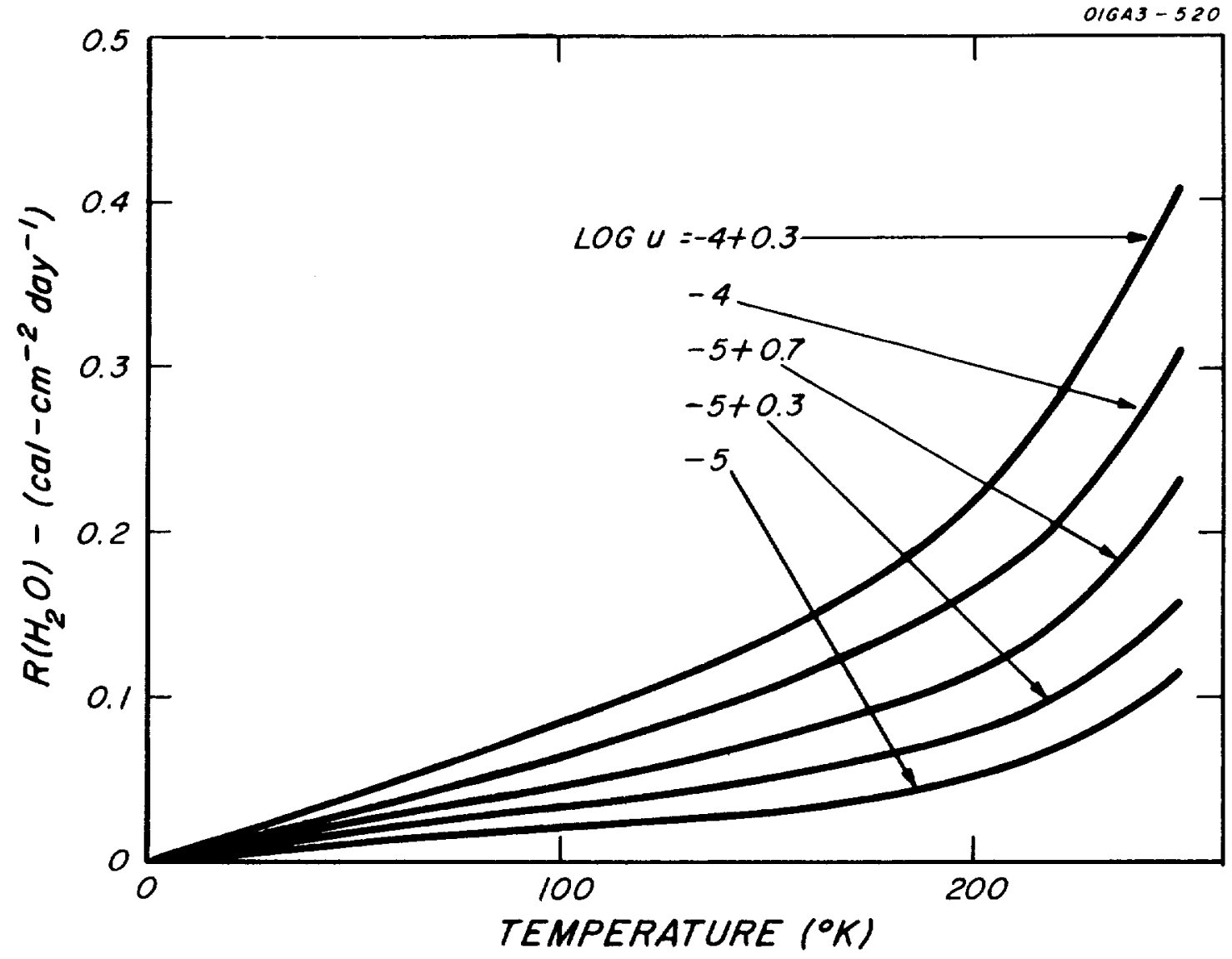

Figure 2. Extrapolated values of $\mathrm{R}\left(\mathrm{H}_{2} \mathrm{O}\right)$. 


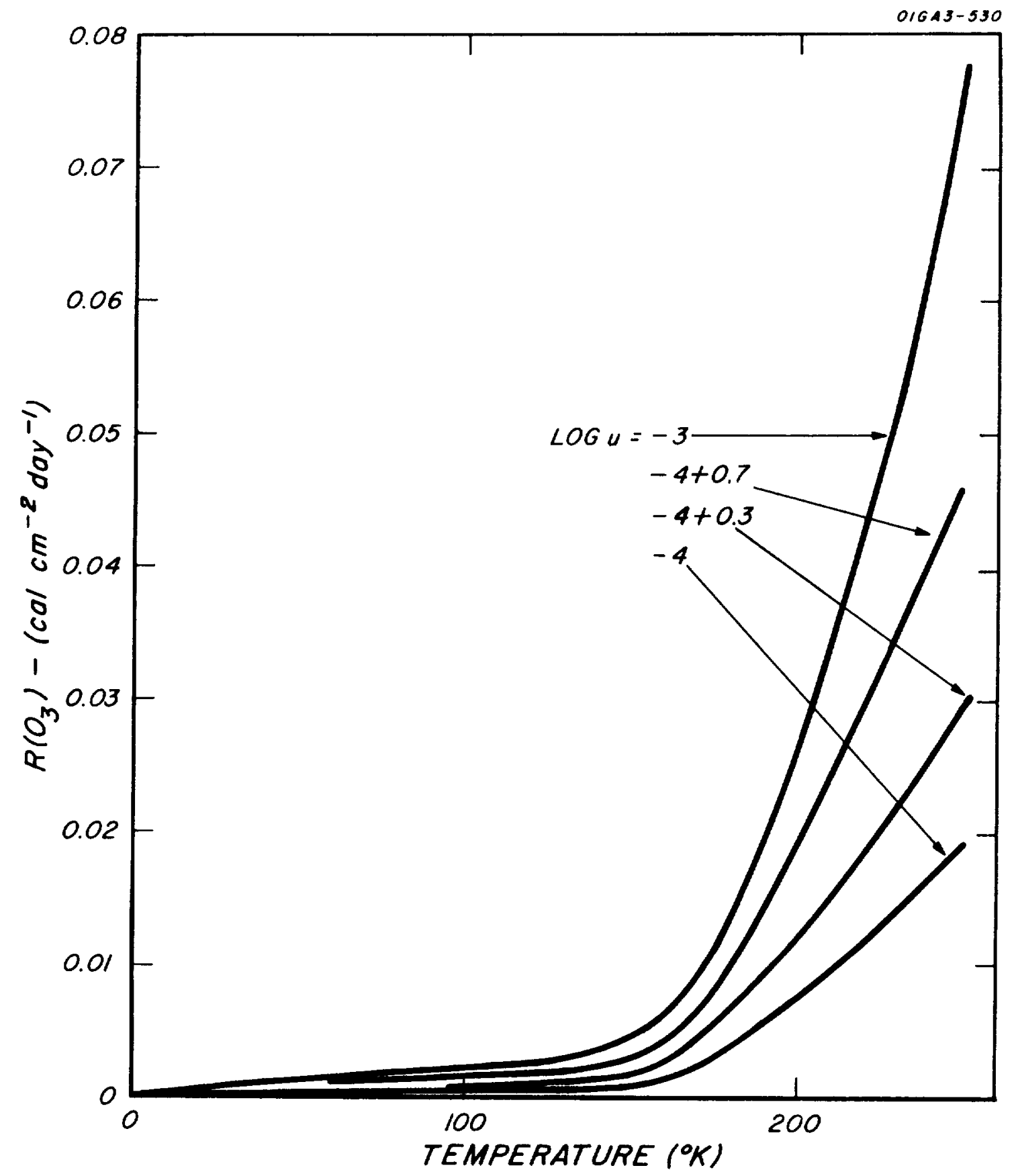

Figure 3. Extrapolated values of $\mathrm{R}\left(\mathrm{O}_{3}\right)$. 


\section{RESULTS}

For a solar constant of $2.0 \mathrm{cal} \mathrm{cm}^{-2} \mathrm{~min}^{-1}$ and a dilution factor of 0.44 , the average incoming radiation at the top of the Martian atmosphere, $\mathrm{I}_{\mathrm{o}}$, is $317 \mathrm{cal} \mathrm{cm}^{-2} \mathrm{day}^{-1}$. With the commonly accepted value of 0.15 for the Martian planetary albedo the average amount of solar radiation remaining after reflection is

$$
I_{0}(1-A)=269 \mathrm{cal} \mathrm{cm}^{-2} \mathrm{day}^{-1}
$$

Using this value and the maximum green-house model discussed above, we obtain from Equation (5) a value of $\mathrm{T}_{\mathrm{S}}$ equal to $233 \mathrm{~K}_{\text {。 }}$ This temperature is our estimate of the maximum possible value of the average surface temperature on Mars。 Comparing $233 \mathrm{~K}$ with our previous estimate of $219 \mathrm{~K}$ for the minimum possible value, we see that even a maximum green-house effect vould raise the surface temperature only about $14 \mathrm{deg} \mathrm{K}$. This maximum green"house effect can be compared to the average green-house effect for the earth. The temperature of the earth's surface, if it had an atmosphere transparent to long wave radiation, can be computed from Equation (1). With an albedo of $0.35, \mu=1$ and a solar constant of $2.0 \mathrm{ca} \mathrm{cm}^{\circ} \min ^{-1}$. Equation (1) yields a temperature of $252 \mathrm{~K}$ for the earth. The observed average temperature of the earth is about $288 \mathrm{~K}$, indicating that the average green-house effect for the earth is about $36 \mathrm{~K}_{2}$ or about $2 \frac{1}{2}$ times as large as the maximum green ohouse effect for Mars. 
Of the three gases contributing to the green-house effect, carbon dioxide is by far the most important as can be seen from the following values of the integrals appearing in Equation (5):

$$
\begin{aligned}
& \int_{T_{t}}^{T_{S}} R\left(\mathrm{CO}_{2}\right) d T=57 \mathrm{cal} \mathrm{cm}^{-2} \mathrm{day}^{-1} \\
& \int_{T_{t}}^{T_{S}} R\left(\mathrm{H}_{2} \mathrm{O}\right) \mathrm{dT}=18 \mathrm{cal} \mathrm{cm}^{-2} \mathrm{day}^{-1} \\
& \int_{T_{t}}^{T_{s}} R\left(O_{3}\right) d T=4 c a 1 \mathrm{~cm}^{-2} \text { day }^{m 1}
\end{aligned}
$$

This is in contrast to the earth ${ }^{2} s$ atmosphere where water vapor is the most important absorber of long-wave radiation.

Another measure of the effectiveness of the green-house is the ratio of outgoing long wave radiation at the top of the atmosphere to the long wave radiation emitted by the surface, which may be termed the infrared transparency of the atmosphere. The lower the infrared transparency, the more effective is the green house. For average conditions on earth the infrared transparency (based on radiation fluxes computed by Landon ${ }^{(9)}$ ) i.s about $57 \%$ for our maximum green house on Mars, the infrared transparency is $77 \%$ 。

Based upon our theoretical estimates the true average surface temperature of Mars should lie between $21.9 \mathrm{~K}$ and $233 \mathrm{~K}$. In order to fix the average temperature more accurately, it would be necessary to know 
the actual amounts and distribution of absorbing gases, and the actual vertical temperature distribution. A sample calculation indicated that the use of the estimated average carbon dioxide concentration - $2 \%$ by volume - rather than a maximum concentration - 4\% by volume - did not affect the outgoing radiation too much. Also the vertical temperature distribution we used cannot be too far from the actual temperature dis. tribution. We therefore suggest that the actual mean temperature is closer to $233 \mathrm{~K}$ than to $219 \mathrm{~K}_{\mathrm{a}}$ In the next section we compare our estimate with some of the observations of Martian temperatures. 


\section{COMPARISON WITH OBSERVATIONS}

The observations of surface temperature on Mars are generally based upon measurements of the planet's thermal emission; these measurements are wovaly made during oppositions and therefore refer largely to the sunlit side of the planet. Gifford ${ }^{(8)}$ ias analyzed systematically large numbers of such observations and has derived average latitudinal surface temperature distributions on the noon meridian for each of the four seasons for the latitude range $60^{\circ} \mathrm{N}$ to $80^{\circ} \mathrm{S}$. On the basis of continuity and solar insolation considerations we have extrapolated these curves to the poles; they are shown in Figure 4, with extrapolations indicated by dashed lines. In order to compute the average planetary surface temperature from these observations we mist perform the following operations:

(1) Average the seasonal curves to obtain an average annual curve;

(2) Subtract from these noon-time temperatures the amplitude of the average diurnal variation of temperature as a function of latitude in order to obtain a mean daily temperature;

(3) Compute the average planetary surface temperature by the area of the latitude belt.

When the first of these operations is performed the average annual noon curve shown in Figure 5 results. The correction of this curve for diurnal variation is important because such variations are apparently 


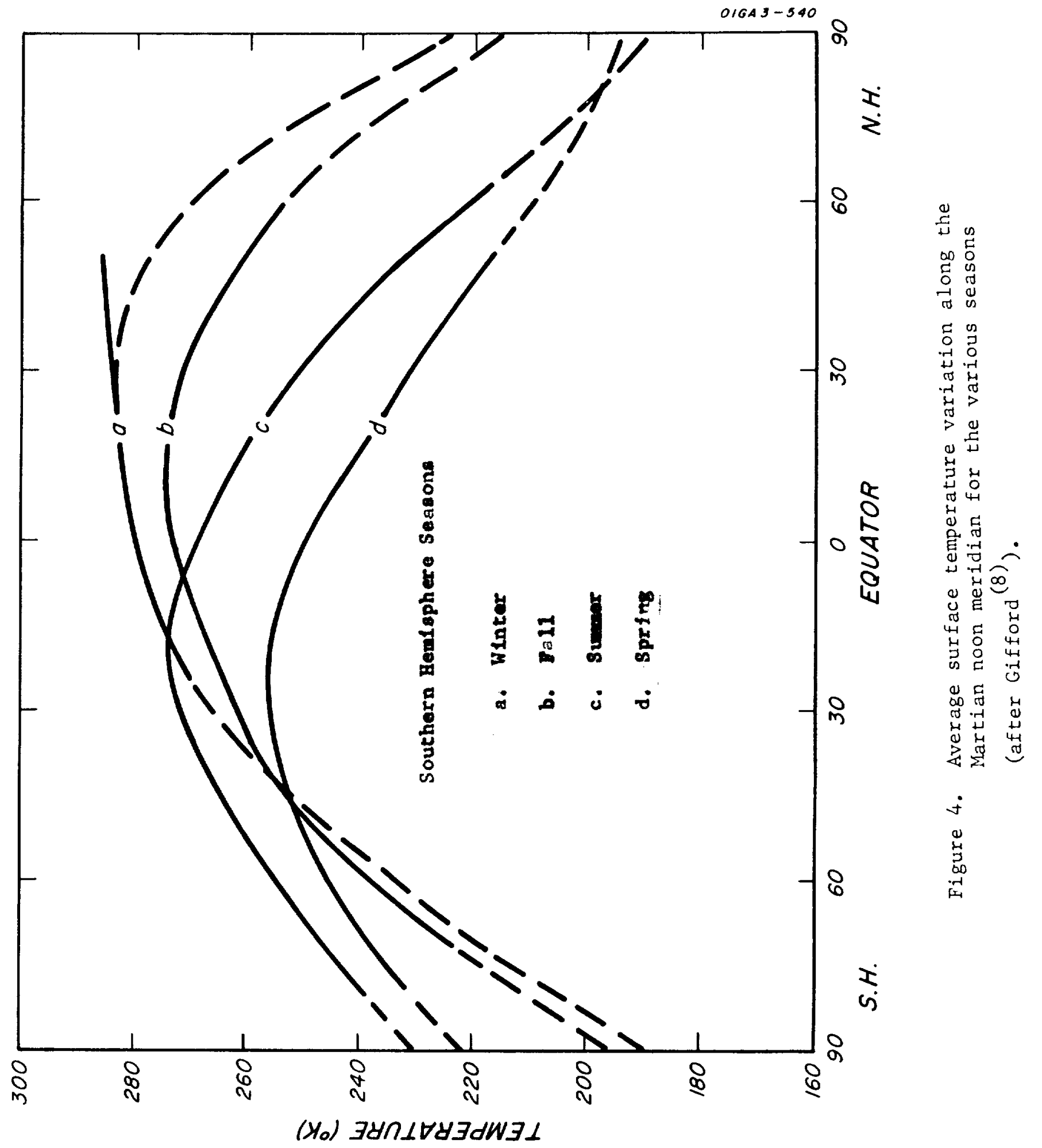




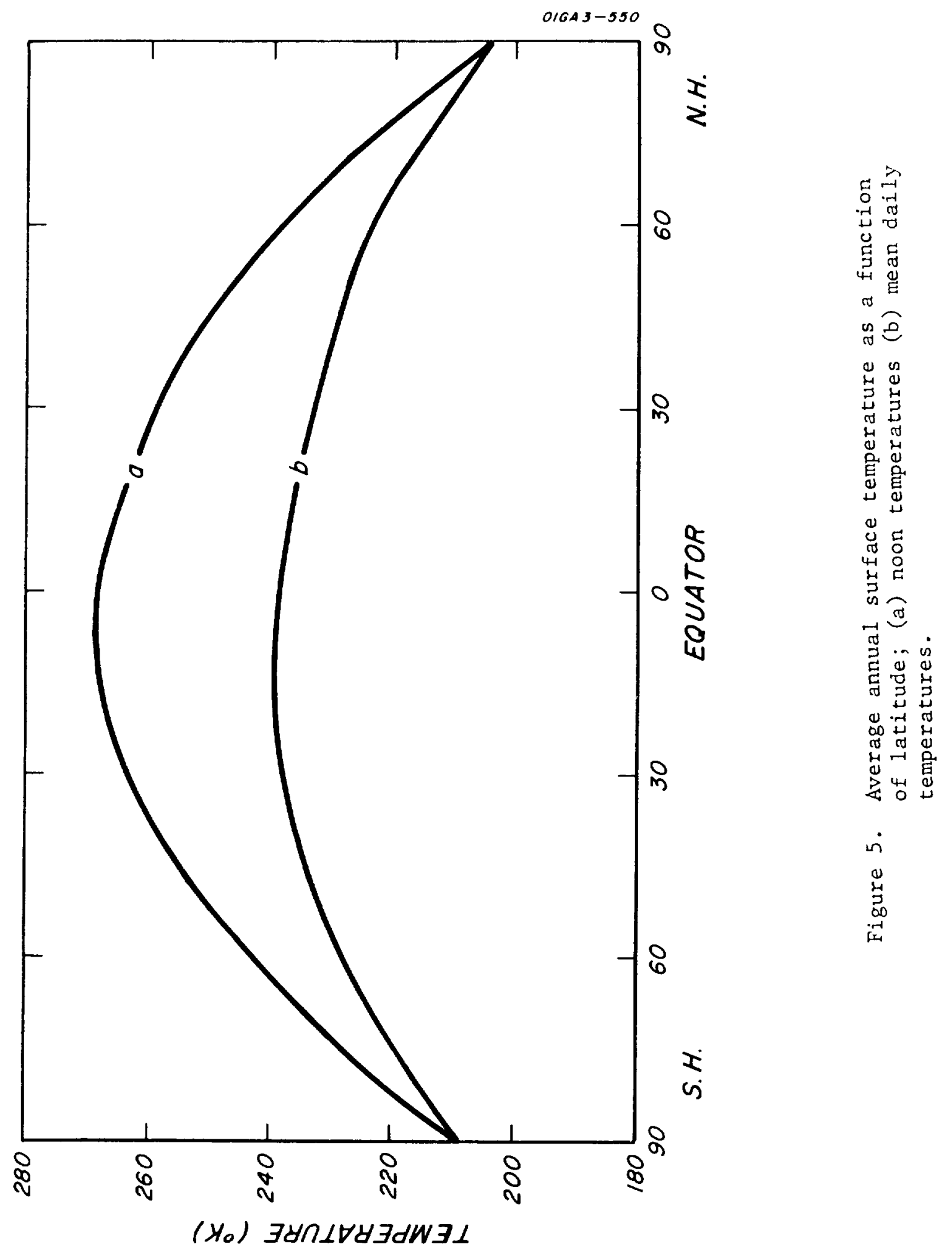


appreciable on Mars. There have been a number of measurements, mostly in tropical regions, of the variations of Martian surface temperature during the day. Gifford's ${ }^{(8)}$ analysis of 214 daytime surface tempera. tures near the Martian equator indicates a diurnal range of about $70 \mathrm{C}$, which is equivalent to an amplitude of $35 \mathrm{C}$. DeVaucouleurs ${ }^{(6)}$ in his book on Mars, suggests an average amp1itude of $25 \mathrm{C}$ in the equatorial regions. In the recent observations of Sinton and Strong ${ }^{(10)}$, however, the indicated amplitude is about $45 \mathrm{C}$ or higher. Since the analyees of Gifford and DeVaucouleurs are based upon more data we shall rely on their estimates for the amplitude of the diurnal variation at the equator; we therefore adopt an amplitude of $30 \mathrm{C}$ in the equatorial regions of Mars. Assuming that the diurnal variation depends only on insolation, we can approximate the latitudinal variation of the amplitude by a simple cosine function

$$
a_{\varphi}=30 \cos \varphi
$$

where $a_{\varphi}$ is the amplitude at any latitude $\varphi_{0}$ When the se amplitudes are subtracted from the noon curve, the average annual curve shown in Figure 5 is obtained. The curve indicates an average equatorial temperature close to $240 \mathrm{~K}$ and average polar temperature of between $200 \mathrm{~K}$ and 210K. The average planetary surface temperature can be obtained from a numerical integration of this curve from pole to fole, weighting tie.e temperztures ascording to surface area as follows:

$$
\overline{\mathrm{T}}=\frac{1}{2} \int_{-\pi / 2}^{\pi / 2} \overline{\mathrm{T}}(\varphi) \cos \varphi \mathrm{d} \varphi
$$


where $\bar{T}$ is the average planetary surface temperature, and $\bar{T}(\varphi)$ is the average surface temperature at latitude $\varphi$. Upon performing this integration we obtain a value of $233 \mathrm{~K}$ for the average Martian surface temperature. Within the scope of the uncertainties involved in obtaining this value, it can be considered to be in reasonable agreement with the computed theoretical range $219 \mathrm{~K}$ to $233 \mathrm{~K}$. 
VI. SUMMARY

From theoretical computations based upon radiative equilibrium considerations, the average surface temperature of the planet Mars is found to be in the range of $219 \mathrm{~K}$ to $233 \mathrm{~K}$.

This is in reasonable agreement with the thermal emission observations which suggest a mean temperature of about $233 \mathrm{~K}$ 。 The computations also indicate that the maximum green-house effect on Mars is about $40 \%$ of the average green-house effect in the earth's atmosphere, and that carbon dioxide is the most important contributor to the Martian green house. 


\section{REFERENCES}

1. Elsasser: $W_{0} M_{0}$, Atmospheric Radiation Tables, Amer. Meteor. Soc. Meteorological Monographs: 4. 23, 43 (1960)。

2. Hales, J, Vo, Williams, To L., Henderson, D., "Calculation of Infrared Radiative flux Emission of the Earth Plus Atmosphere at Various Levels High tive the Earth"' Final Report Contract AF $19(604)-2418$. (1960).

3. Grandjean: J., and Goody, R. $M_{0}$, "The Concentration of Carbon Dioxide in the Atmosphere of Mars", Ap. J., 121. 548 (1955).

4. Kuiper, G. P., The Atmospheres of the Earth and Planets, Univ。 of Chicago Press, 434 (1952)。

5. Dunham $T_{0}$ "Spectroscopic Observations of the Planets at Mount Wilson", The Atmospheres of the Earth and Planets, Ed。 by G。 P. Kuiper: 288=305 (1952).

6. DeVaucouleurs. Go Physics of the Planet Mars, Faber and Faber Limited, London, 365 (1954).

7. Marmo, $F_{0} F_{0}$, Watanabe, $K_{0}$, Dalgarno, A., Warneck, P. , "Laboratory and Theoretical Studies in the Vacuum Ultraviolet for the Investigation of Chemical Physics of Planetary Atmospheres", Third Quarter1y Progress Report, Contract No. NASw-124, GCA, Bedford,
Masso, 70 (1960).

8. Gifford, $F_{2}$, "The Surface Temperature Climate of Mars", Ap. J, 123, 154-161 (1956)。

9. Landon, $\mathrm{J}_{\text {: }}$ "A Study of the Atmospheric Heat Balance", Final Report Contract No. AF 19 (122)-165, AD 117227, Dept. of Meteorology and Ocenography, N.Y.U., 99 (1957).

10. Sinton, $W_{0} M_{0}$, and Strong, J. "Radiometric Observations of Mars", Ap. $\mathrm{J}_{0}=131$, $459-469$ (1960)。 